EXPERIMENTAL INFECTION OF GIRAFFE (Giraffa camelopardalis) WITH SAT-1 AND

\title{
SAT-2 FOOT AND MOUTH DISEASE VIRUS
}

W. VOSLOO ${ }^{1,2^{*}}$, S.P. SWANEPOEL ${ }^{1,3}$, M. BAUMAN ${ }^{1}$, B. BOTHA ${ }^{1}$, J.J. ESTERHUYSEN ${ }^{1}$, C.I. BOSHOFF $^{1,4}$, D.F. KEET ${ }^{5}$, A. DEKKER ${ }^{5}$

${ }^{1}$ Transboundary Animal Diseases Programme, ARC-Onderstepoort Veterinary Institute, Private Bag X05, Onderstepoort, 0110, South Africa

${ }^{2}$ Department of Tropical Veterinary Diseases, Faculty of Veterinary Medicine, University of Pretoria, South Africa

${ }^{3}$ Deltamune, PO Box 14167, Lyttelton, 0140, South Africa

${ }^{4}$ Department of Biomedical Sciences, Tshwane University of Technology Private Bag X680, Pretoria, 0001, South Africa

${ }^{5}$ Office of the State Veterinarian, Kruger National Park, P.O. Box 12, Skukuza, 1350, South Africa

*Corresponding author, Wilna.vosloo@csiro.au

Current address: Australian Animal Health Laboratory, Private Bag 24, Geelong, 3220, Australia

Number of tables: 1

Number of figure: 2

Field work performed at 5, laboratory assays performed at 1

Running head: foot-and-mouth disease virus, SAT types, giraffe 


\section{SUMMARY}

The potential role of giraffe (Giraffa camelopardalis) in the epidemiology and spread of foot and mouth disease SAT types was investigated by experimental infection and detection of virus in excretions using virus isolation on primary pig kidney cell cultures. In two experiments separated by a period of 24 months, groups of four animals were needle infected with a SAT-1 or SAT-2 virus respectively and two in-contact controls were kept with each group. Viraemia was detected 3-9 days post infection, and from mouth washes and faeces only occasionally up to day 13 . The SAT-1 virus was transmitted to only one in-contact control animal, probably via saliva that contained virus from vesicles in the mouth of a needle-infected animal. None of the animals infected with the SAT-2 virus had any vesicles in the mouth and there was no evidence of transmission to the in-contact controls. No virus was detected in probang samples for the duration of the experiments (60 days post infection), indicating that persistent infection probably did not establish with either of these isolates. Giraffe most likely do not play an important role in FMD dissemination. Transmission of infection would possibly occur only during close contact with other animals when mouth vesicles are evident.

\section{KEYWORDS}

Foot and mouth disease, SAT types, giraffe, infection, virus isolation, primary pig kidney cells

\section{INTRODUCTION}

Foot and mouth disease (FMD) is caused by single-stranded, positive sense RNA viruses that have been shown to infect over 70 species of cloven-hoofed animals (Hedger, 1981). Wildlife such as the African buffalo (Syncerus caffer) are important in the maintenance and spread of the SAT-serotypes, which complicates the control of FMD in Africa. However, apart from a limited number of species, the involvement of other free-living species in the maintenance and spread of SAT-serotype infections remains unclear. This uncertainty results in regulatory authorities instituting control strategies which may be considered by some to be over-zealous. That, in turn, renders the translocation and utilization of many wildlife species problematic and complicates rural development based on wildlife-domestic 
livestock integration. The lack of information also has the effect of limiting access to international markets for products derived from livestock and wildlife (Perry and Randolph, 2003; Thomson et al., 2003b; Vosloo and Thomson, 2004).

African buffalo are the only wildlife species which has been shown to be capable of maintaining the SAT-serotypes of FMD virus for extended periods of time, i.e. for periods of several years (reviewed by Thomson and Bastos, 2004; Vosloo and Thomson, 2004). It appears that, in southern Africa at least, buffalo are by far the most common source of infection for other wild and domestic species, where they come into close contact (Bastos et al., 2000; Vosloo et al., 2002; 2006).

Control of FMD in southern Africa is reliant on separation of potentially infected wildlife from susceptible domestic animals using fencing, and control of the movement of susceptible animals and animal products. Susceptible cattle populations are often vaccinated (Brückner et al., 2002; Thomson et al., 2003a). However, more refined management strategies that do not unnecessarily restrict conservation and commercial exploitation of wildlife resources are needed. The strategies must take account of the importance of rural development in many parts of sub-Saharan Africa where wildlife is a valuable resource. A better understanding of the role that different species of free-living wildlife play in the epidemiology of FMD is necessary in order to develop more efficient FMD management strategies and policies in southern Africa specifically.

Apparent clinical disease supported by sero-positive results has been documented in giraffe (Giraffa camelopardalis) and serologically positive animals have been found in the Kruger National Park (KNP) (Records of the Transboundary Animal Diseases Programme (TADP), ARC-Onderstepoort Veterinary Institute and State Veterinarian, KNP). However, their susceptibility to infection and ability to propagate SAT-serotype infections is unknown. This study aimed to investigate the levels of virus excreted by acutely infected giraffe using virus isolation and the persistence of the virus in the animals after recovery from infection. 


\section{MATERIALS AND METHODS}

\section{Infection experiments}

The experiments were conducted strictly according to the ethics requirements of the ARCOnderstepoort Veterinary Institute and were also approved by the South African National Parks conservation and ethics committees. Two groups of six sub-adult giraffe each ranging in age between 14 and 24 months were used. The giraffe were captured and kept in a quarantine area in open pens in the endemically infected KNP and tested for antibodies to the three SAT types at the onset of the experiment. Four of the six animals were infected by an intramuscular injection of $1 \mathrm{ml}$ virus into the gluteal muscles and $0.1 \mathrm{ml}$ into the labial gingiva of the lower lip. The remaining two were kept as in-contact controls. One group was infected with a SAT-1 virus previously isolated from impala in the north of the KNP $\left(\mathrm{SAR} 9 / 81\left[\mathrm{BTY}_{3} \mathrm{BHK}_{3} \mathrm{~B}_{1} \mathrm{BHK}_{4} \mathrm{~B}_{1}\right]\right.$ ) with a titre of $\log _{10} 7.0 \mathrm{CID}_{50}$ (cattle infectious dose) and a SAT-2 virus from buffalo (KNP19/89/2 [PK1B1]) at a titre of $\log _{10} 6.0 \mathrm{CID}_{50}$. Both viruses were adapted to and titrated in cattle prior to infecting the giraffe. The SAT-1 and SAT2 infections were performed 24 months apart to preclude cross contamination.

\section{Sample collection for virus detection}

Samples from each giraffe, including the controls, were collected at three-day intervals or longer (one giraffe per day), from 3-18 days after inoculation and thereafter 30 and 60 days after infection. The staggered 3-day sampling schedule was instituted to ensure that the animals had sufficient time for recovery between chemical immobilisations. They were immobilized with a combination of etorphine HCL and azaperone as described by Morkel in the Capture \& Care Manual (Morkel, 1993). Blood in EDTA and serum samples were collected from the jugular veins. Nasal and genital swabs were taken from the nasal passage and the preputial opening or the vestibulum of the vagina respectively using swabs placed in $5 \mathrm{ml}$ phosphate buffered saline (PBS pH7.4). Faeces ( $\square 1 \mathrm{~g}$ ) were extracted from the rectum with a gloved finger, placed in $10 \mathrm{ml}$ PBS and the supernatant recovered. Mouth washes were performed by inoculating $10 \mathrm{ml}$ PBS into the mouth using a syringe and recovering as much liquid as possible. Oesophageal/pharyngeal $(\mathrm{O} / \mathrm{P})$ specimens were collected using the method described by Sutmoller and Gaggero (1965) and the sample collected placed in liquid nitrogen immediately after 
collection. Samples of epithelial lesions were collected when present and placed in PBS. Tonsil, retromandibular, parotid, pharyngeal, haemal and hepatic lymph nodes were collected in PBS from giraffe 2.5 on day 5 when it died.

\section{Virus isolation on cell cultures}

Virus isolation was performed using primary pig kidney cell cultures according to standard procedures. If no cytopathic effect (CPE) was observed after 48 hours, one blind passage was performed and the sample was recorded as negative if no CPE was observed after a further 48 hour incubation. Virus levels were expressed as $\log _{10} \mathrm{TCID}_{50} / \mathrm{ml}$.

\section{Serological determination of neutralising tires}

Neutralising antibodies to SAT-1, SAT-2 and SAT-3 were measured using the assay described by Esterhuysen et al. (1985) and antibody levels are expressed as the reciprocal of the $50 \%$ end-point dilution.

\section{RESULTS}

\section{Response of giraffe to needle infection with SAT-1 and -2 viruses}

With one exception (giraffe $2.1 ; 1.8$ to SAT-2), all the giraffe were negative $(>1.6)$ for neutralizing antibodies to the three SAT serotypes at the start of the experiment The SAT-2 inoculum was administered to this animal to determine whether the antibodies present would protect against infection. It developed lesions 4-5 days later than the other needle inoculated animals (Table 1). Typical FMD lesions were observed as early as 2 days after infection with SAT-1. All eight giraffe inoculated with SAT serotypes 1 or 2 developed distinct FMD lesions on the feet. With SAT-2 infection, the lesions were confined to the interdigital cleft and were large, up to $7 \mathrm{~cm}$ in length. A similar response was seen with the SAT-1 infection but lesions were smaller. Small ruptured vesicles were also found on the coronary band in some of the giraffe. By 30 days after infection the interdigital lesions had completely healed and growth arrest lines were present parallel to the coronary band that moved distally as the hooves continued to grow (Fig. 1).

Typical mouth lesions were only seen in the SAT-1 infection experiment. Small vesicles developed at the site of injection into the gingiva. Vesicles ranging in size from 2 to $10 \mathrm{~cm}$ were also observed on 
the dorsum of the tongue of each of the infected animals (Fig. 2). While these lesions were present the giraffe exhibited hypersalivation, champing and grinding of the teeth, and frequent flicking of the tongue. None of the SAT-2 infected animals developed any mouth lesions, even at the injection site. One of the female giraffe developed vaginitis 7 days post infection, but no virus could be isolated from the vaginal swabs (results not shown).

\section{Transmission of FMD virus from acutely infected giraffe to in-contact giraffe}

One of the two in-contact giraffe (giraffe 1.1) became infected with SAT-1 and developed clinical signs, similar in distribution and extent to the inoculated animals, 15 days after the inoculation of the other 4 giraffe and 10 days after the first appearance of virus in the excretions of the infected animals (Table 1). The other in-contact giraffe (1.5) did not develop clinical signs of FMD and remained serologically negative to SAT-1 at the termination of the experiment (Table 1). This animal had a titre of 1.8 to SAT-2 at day 28 , but this is probably a non specific result as this was the only occasion on which measurable antibody was detected. Neither of the two in-contact giraffe included in the SAT-2 infection experiment ( 2.3 and 2.6) developed clinical signs, no virus was isolated from any of the samples collected and they were sero-negative for SAT-2 at the termination of the experiment (day 60; Table 1). A transient antibody response was detected in one giraffe (2.3) to SAT-2 on day 10 only.

\section{Virus isolation and serological responses}

Virus was isolated from the epithelium collected from mouth and feet vesicles in all cases (not shown). No virus was isolated from any of the probang samples. In the SAT-1 experiment viraemia was evident in 2 of the infected giraffes (Table 1; nos 1.4 and 1.6) on days 4 and 3 respectively. Virus was most consistently isolated from mouth washes between days 5-8 and in one animal, levels reached $10^{4} \mathrm{TCID} / \mathrm{ml}$. One in-contact animal also had virus present in the mouth wash at day 15 (giraffe 1.1), but was negative for virus on all prior sampling occasions, i.e. at days 7 and 11. Seroconversion occurred between days 3 and 8 in the experimentally infected animals $(1.2,1.3,1.4$ and 1.6) and on day 15 in one in-contact animal (1.1) and persisted until the termination of the experiment at day 60. 
In the SAT-2 experiment viraemia in the infected animals was observed between 4-6 days after inoculation, except for the animal that had a titre of 1.8 at the onset of the experiment (giraffe 2.1), where viraema was evident only on day 11 . Virus was isolated from faeces on two occasions 9-13 days after infection in giraffe 2.4 only. Sero-conversion was evident from day 8 onwards and antibodies to SAT-2 remained high for the duration of the experiment (i.e. until day 60). One of the giraffe (number 2.5) died 5 days after the commencement of the experiment as a result of complications with immobilisation but was sero-negative at that time. Samples from this animal were taken during the post-mortem examination and virus was isolated from the tonsil, retromandibular lymph node and parotid lymph node but not from the other samples (Table 1).

\section{Discussion}

Most experiments involving the SAT type viruses in wildlife have been limited to inoculation of a few animals with a limited range of virus isolates and results should be interpreted with caution. Since wild animals have to be immobilised for sampling, it is not possible to sample individual animals at frequent intervals. It is also known that virus excretion differs between animals, depending on the dose of virus administered, the route of infection and the virus isolate used. However, this experiment adds to our limited understanding of FMD in giraffe and their potential role in the epidemiology of the disease in sub-Saharan Africa.

The clinical signs observed in giraffe were similar to those observed in other wildlife species with mouth lesions and lesions around the coronory band and interdigital spaces. However, there were differences between the two serotypes, with mouth lesions completely absent in the SAT-2 experiment. Although lameness has on occasion been observed in giraffe during some natural outbreaks of FMD in the KNP, it was not evident in these experiments, probably due to the flooring conditions provided to the captive giraffe (thick layer of river sand). Recovery from clinical disease was rapid. A break in the surface of the hoof wall and sero-conversion were the only indications of previous disease when the experiments were terminated after 60 days. Persistent infection of the oropharyngeal mucosa was not demonstrated, i.e. all probang samples tested were negative for infectious virus on cell culture. However, as far as our knowledge goes probang sampling in giraffe has not 
been attempted before, and it is therefore possible that the technique was at fault or that virus replication sites are different in giraffe.

Based on these data, it seems that viraemia in giraffe started between 3 and 4 days after needle infection and lasted until day 9 in one individual. In cattle and African buffalo infected with SAT-1 and SAT-2 isolates, viraemia was detected 1-5 days after infection (Gainaru et al., 1986). However, due to ethical considerations, giraffe could only be handled every third day, and it is possible that viraemia could have started earlier in some animals. The challenge viruses were cattle-adapted which could impact on virus replication in a new host. In addition, primary pig kidney cells were used for isolation, and although shown to be the most sensitive for isolations from various species in our hands, could have had an impact on the results. Titres during viraemia in giraffe ranged between 1.2 and $3.5 \log _{10} \mathrm{TCID} / \mathrm{ml}$ which were lower than the titres observed in some cattle and buffalo infected with SAT viruses, also determined using primary pig kidney cells $\left(10^{1.3}\right.$ and $\left.10^{4.8} / \mathrm{ml}\right)$ (Gainaru et al., 1986).

Virus was only detected in the mouth washes of giraffe infected with SAT-1 and where mouth lesions were observed. The virus probably originated from vesicular fluid of ruptured vesicles, since the animals infected with SAT-2 had no mouth lesions and no detectable virus in the mouth wash. The virus titres in mouth washes varied between 1.2 and $4 \log _{10} \mathrm{TCID} / \mathrm{ml}$ and lasted up to 6 days, except in one in-contact animal (giraffe 1.1) where it was detected at day 15, probably due to the later onset of disease (Table 1). Similar titres were observed in the saliva of cattle and buffalo infected with SAT type viruses, but the cattle had mouth lesions while the buffalo did not (Gainaru et al., 1986). The presence of virus in the mouth wash in the infected giraffe was most probably the source of infection for the in-contact animal, as no transmission occurred with the SAT-2 infected giraffe where no mouth lesions or virus in washes were observed. Transmission under these circumstances seems to be inefficient, as only one of the in-contact animals became infected with SAT-1, and none with SAT2. Virus was isolated from faeces from giraffe 2.4 in the SAT-2 experiment at low levels that lasted up to 13 days after infection. Although faecal excretion has also been found in acutely infected buffalo, it is probably a rare occurrence, while in cattle no SAT-1 and SAT-2 virus was found in faeces 
(Gainaru et al., 1986). Excretion of virus in faeces may serve as a source of virus in the field, but the levels detected were low and, because faecal balls dry quickly, any virus present would probably be rapidly inactivated.

The low antibody level to SAT-2 detected in one giraffe prior to the commencement of the experiment appeared to confer some protection because viraemia in this animal was delayed for at least 5 days compared to the other needle-infected cohorts. However, the animal did finally succumb to clinical disease. The transient heterologous serological reactions of one animal in the SAT-1 and one in the SAT-2 experiment could be due to non-specific reactions, as these were observed for only one sampling period. All the other animals still had high antibody levels 60 days after infection. It is also possible that these could be an anamnestic response to previous infection where antibody levels have waned.

This limited study shows that giraffe are susceptible to FMD virus infection and although this study was based on a small number of animals and only 2 virus serotypes, it seems unlikely that they play an important role in maintaining and spreading the infection in the wild.

\section{REFERENCES}

Bastos, A.D.S, C.I. Boshoff, D.F. Keet,, R.G. Bengis, and G.R. Thomson, 2000: Natural transmission of foot-and-mouth disease virus between African buffalo (Syncerus caffer) and impala (Aepyceros melampus) in the Kruger National Park, South Africa. Epid. Infect. 124, 591-598.

Brückner, G.K., W. Vosloo, B.J. Du Plessis, P.E. Kloeck, L. Connoway, M.D. Ekron, D.B. Weaver, C.J. Dickason, F.J. Schreuder, T. Marais, and M.E Mogajane, 2002: Foot and mouth disease: the experience of South Africa. Rev. Sci. Tech. 21, 751-64.

Esterhuysen, J.J., G.R. Thomson, J.R. Flammand, and R.G. Bengis, 1985: Buffalo in the northern Natal game parks show no serological evidence of infection with foot-and-mouth disease virus. Onderstepoort J. Vet. Res. 52, 63-66.

Gainaru, M.D., G.R. Thomson, R.G. Bengis, J.J. Esterhuysen, W. Bruce, and A. Pini, 1986: Footand-mouth disease and the African buffalo (Syncerus caffer). II. Virus excretion and transmission during acute infection. Onderstepoort J. Vet. Res. 53, 75-85. 
Hedger, R.S., 1981: Foot-and-mouth disease. In: Davis, J.W., L.H. Karstad, and D.O. Trainer (eds), Infectious Diseases of Wild Mammals 2nd edn. pp. 87-96. Iowa State University Press.

Morkel, P., 1993: Chemical capture of the giraffe Giraffa camelopardalis. In: McKenzie, A. (ed), The Capture and Care Manual - capture, care accommodation and transportation of wild African animals, pp. 601-610. Published by Wildlife Decision Support Services and the South African Veterinary Foundation.

Perry, B.D., and T.F. Randolph, 2003: The economics of foot and mouth disease, its control and its eradication. In: Foot and mouth disease control strategies. Bodet, B., and M. Vicari (eds), pp. 23-41. Elsevier SAS, Paris,

Sutmoller, P., and A. Gaggero, 1965: Foot-and mouth diseases carriers. Vet. Rec. 77, 968-969. Thomson, G.R., and A.D.S. Bastos, 2004: Foot-and-mouth disease. In: Coetzer J.A.W., and R.C. Tustin (eds.), Infectious Diseases of Livestock, $2^{\text {nd }}$ edn. pp. 1324-1365. Oxford University Press, South Africa.

Thomson, G., B. Dungu, K. Tounkara, W. Vosloo, A. Bastos, and K. Bidjeh, 2003a: Suitability of currently available vaccines for controlling the major transboundary diseases that afflict sub-Saharan Africa. Dev. Biol. (Basel). 114, 229-241.

Thomson, G.R., W. Vosloo, and A.D.S. Bastos, 2003b: Foot and mouth disease in wildlife. Virus Res. 91, 145-161.

Vosloo, W., A.D.S. Bastos, and C. Boshoff, 2006: Retrospective genetic analysis of SAT-1 type footand-mouth disease outbreaks in southern Africa. Arch. Virol. 151, 285-298.

Vosloo, W., and G.R. Thomson, 2004: Natural habitats in which foot and mouth disease viruses are maintained. In: Foot-and-mouth disease: current perspectives. Domingo, E., and F. Sobrino (eds), pp. 383-410. Horizon Bioscience, Great Britain.

Vosloo, W., K. Boshoff, R. Dwarka, and A. Bastos, 2002: The possible role that buffalo played in the recent outbreaks of foot-and-mouth disease in South Africa. Ann. NY Acad. Sci. 969, 187-90. 
Legends to figures:

Table 1

Summary of the positive virus isolation and serological results in giraffe infected with a SAT 1 and SAT 2 virus respectively

Figure 1

Feet lesions observed in giraffe infected with SAT-1.

a) Caudal view of the coronary band at 7 days post infection

b) Caudal view of the coronary band at 28 days post infection

c) Coronary lesions at 7 days post infection

d) Coronary lesions at 42 days post infection

e) Interdigital ruptured vesicle 7 days post infection

f) Coronary lesions in a field case of FMD infection estimated to be $28-36$ days old

Figure 2

Tongue lesions observed in giraffe infected with SAT-1

a) Vesicles observed on the dorsal side of the tongue 4 days post infection

b) Sloughing of tongue epithelium 6 days post infection

c) Sloughing of tongue epithelium 8 days post infection

d) Sloughing of tongue epithelium 10 days post infection 
Table 1 . Summary of the positive virus isolation and serological results in giraffe infected with a SAT 1 and SAT 2 virus respectively

\begin{tabular}{|c|c|c|c|}
\hline SAT-1 infection & $\begin{array}{l}\text { Day first } \\
\text { sampled }\end{array}$ & Virus isolation and titre $\left(\log _{10} \mathrm{TCID} / \mathrm{ml}\right)$ & $\begin{array}{l}\text { Sero- } \\
\text { conversion }\end{array}$ \\
\hline Giraffe 1.1 (in contact) & Day 7, 11 & Day 15: mouth wash (1.2) & Day 15 \\
\hline Giraffe 1.2 & Day 5 & Day 5: mouth wash (3.5) & Day 5 \\
\hline Giraffe 1.3 & Day 6 & Day 6: mouth wash (4.0), coronary skin (1.7) & Day 6 \\
\hline Giraffe 1.4 & Day 4 & Day 4: plasma (3.0); Day 8: mouth wash (3.0) & Day 8 \\
\hline Giraffe 1.5 (in contact) & Day 9 & None & Day 28 (SAT-2)* \\
\hline Giraffe 1.6 & Day 3 & Day 3: plasma (1.2) & Day 3 \\
\hline \multicolumn{4}{|l|}{ SAT-2 infection } \\
\hline Giraffe $2.1^{\#}$ & Day 3, 7 & Day 11: plasma (3.5), Day 14: vesicle (1.7) & Day 0 \\
\hline Giraffe 2.2 & Day 4 & Day 4: plasma (2.2), Day 8: interdigital epithelium & Day 8 \\
\hline Giraffe 2.3 (in contact) & Day 11 & None & Day $10^{*}$ \\
\hline Giraffe 2.4 & Day 6 & Day 6: plasma (3.2), Day 9: plasma (1.6), faeces (1.6), Day 13: faeces (1.0) & Day 9 \\
\hline Giraffe $2.5^{* *}$ & Day 5 & $\begin{array}{l}\text { Day 5: interdigital skin (4.2), tonsil (1.2), retromandibular lymph node (3.2), } \\
\text { parotid lymph node }(1.7)^{\$}\end{array}$ & None \\
\hline Giraffe 2.6 (in contact) & Day 11 & None & None \\
\hline
\end{tabular}

* Each animal had a titre of $\log _{10} 1.8$ to SAT- 2 on a single occasion and both were serologically negative at all other samplings ${ }^{\#}$ Animal had a titre of $\log _{10} 1.8$ to SAT-2 at the onset of the experiment

** Animal died at day 5

\$ plasma, mouth wash nasal swab, genital swab, faeces, pharyngeal lymph node, haemal lymph node, hepatic lymph nodes were all negative for virus isolation 


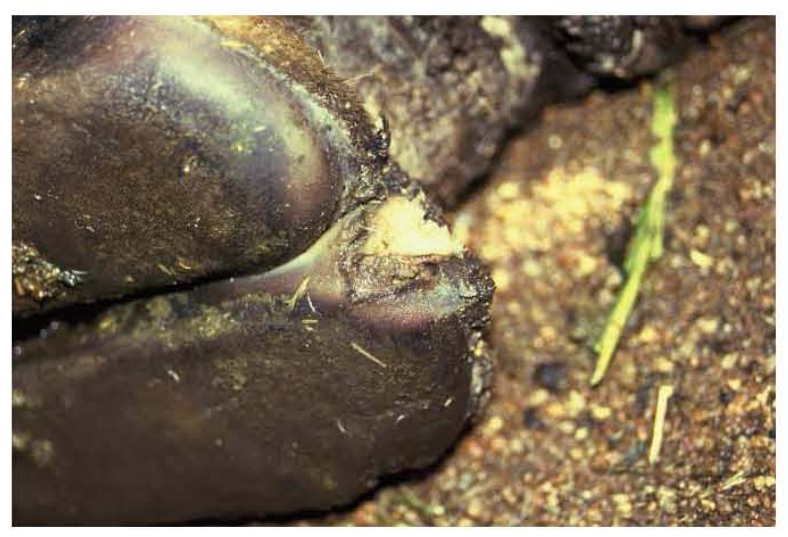

Fig 1a

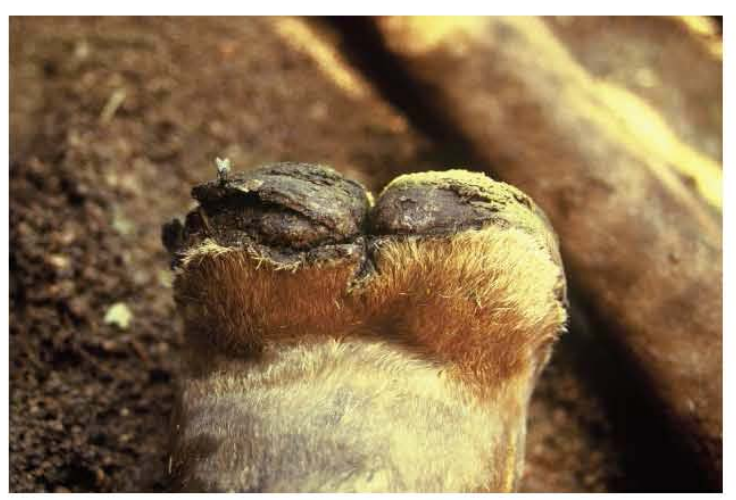

Fig $1 b$

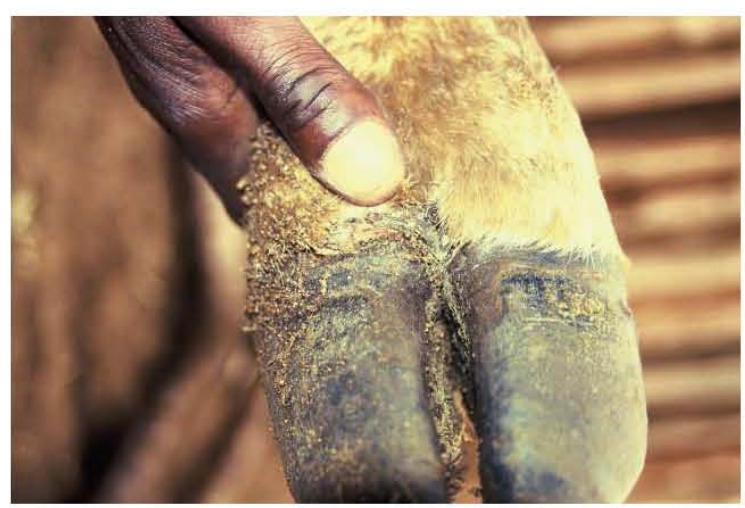

Fig $1 c$

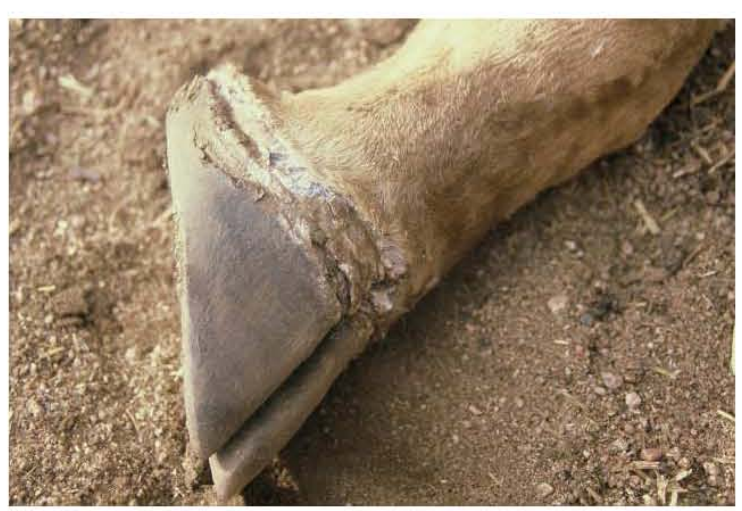


Fig 1d

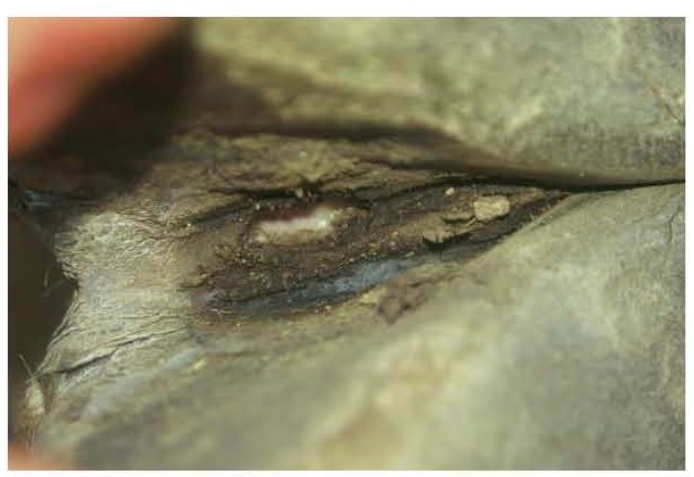

Fig $1 \mathrm{e}$

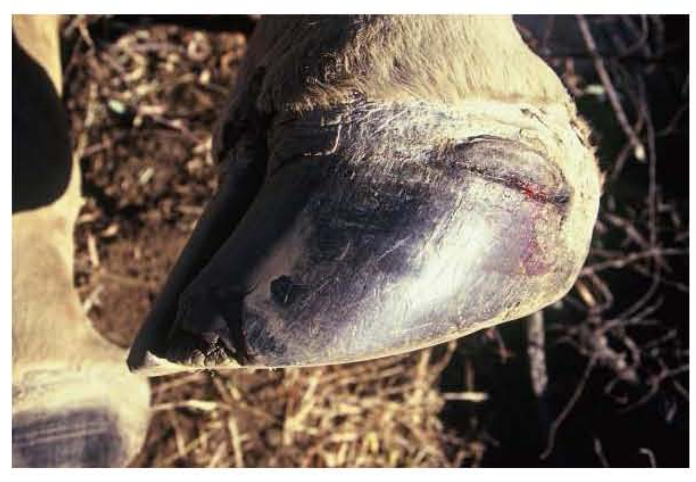

Fig $1 f$ 


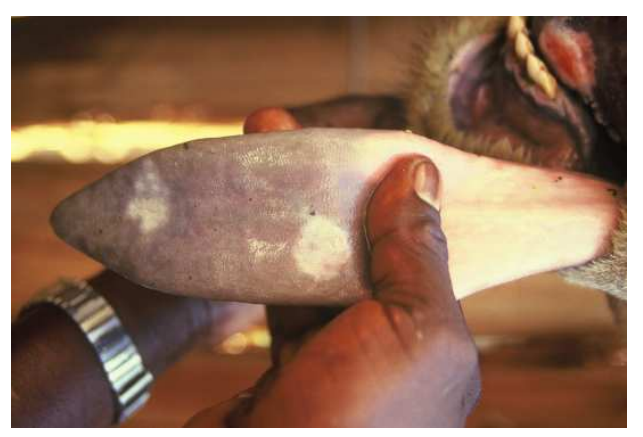

Fig 2a

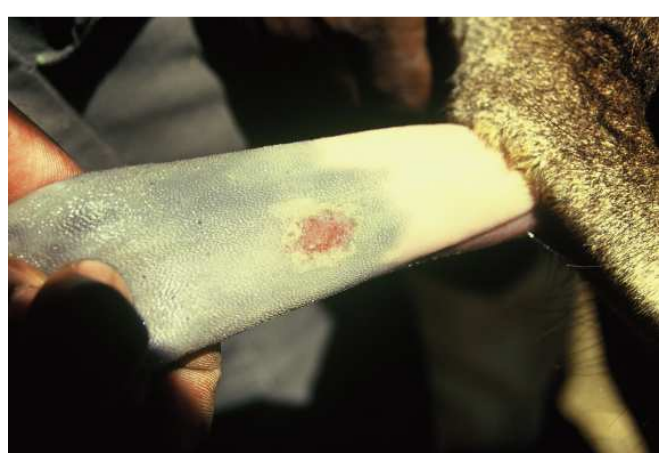

Fig $2 b$

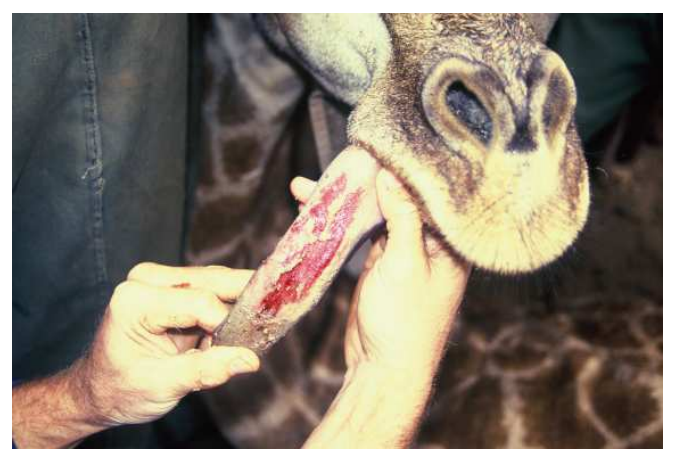

Fig 2c

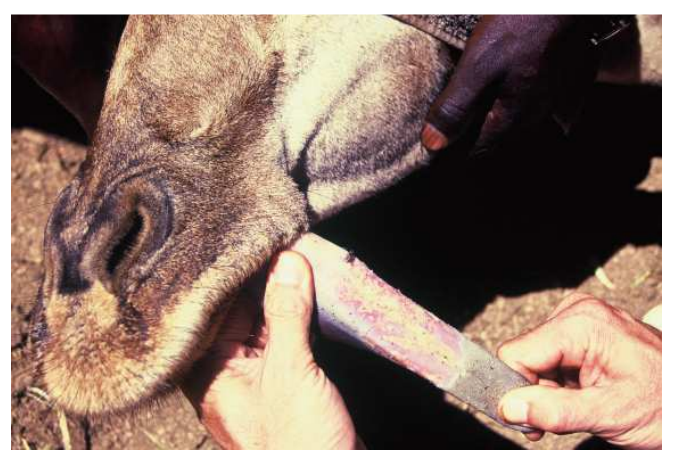

Fig $2 d$ 